\title{
The Phonetic Exponency of Phrasal Accentuation in French and German
}

\author{
William Barry, Bistra Andreeva, Ingmar Steiner \\ Institute of Phonetics, Saarland University, Germany \\ wbarry@coli.uni-sb.de, andreeva@coli.uni-sb.de, steiner@coli.uni-sb.de
}

\begin{abstract}
The acoustic-phonetic properties of words spoken with three different levels of accentuation (de-accented, pre-nuclear and nuclear accented in broad-focus and nuclear accented in narrow-focus) are examined in question-answer elicited sentences and iterative imitations (on the syllable $d a$ ) produced by six French and six German speakers. Normalised parameter values allow a comparative weighting of the properties employed in differentiating the three levels of accentuation. Clear differences are found between French and German in the weighting hierarchy of the acoustic properties.

Index Terms: phrasal accentuation, broad and narrow focus, duration, fundamental frequency, spectral balance, formants
\end{abstract}

\section{Introduction}

Most languages employ prominence-giving mechanisms to highlight the informational importance of particular words in a phrase (e.g., topic accent, focus accent). At some basic production level, the speaker invests more effort in these (accentuated) words, and the observed acoustic effects are greater duration and intensity, higher or changing fundamental frequency $\left(\mathrm{F}_{0}\right)$; and in some way more distinct spectral properties. Some of the change in these properties can be considered a universal, physically determined response to greater effort. But the phonological structure of all language is universally constrained by the common production apparatus delivered at birth, and phonologies still vary greatly from language to language. Thus, the degree to which each of the four parameters underlying prominence are used to signal prominence may be expected to vary between languages. The variation is likely to be a function of the degree to which they are required for other parts of the phonology.

In this study, we examine the production of different accentuation levels in French and German, our basic presumption being that the control of accentuation is subject to language-specific strategies. An immediate implication is that this interferes with our production (and perception [1]) of accentual prominence in another language. L2 production of German by French learners (and vice versa) clearly reveals interference in terms of prominence patterns (i.e., rhythm). But it is unclear whether this is due to different weighting of prominence-supporting parameters rather than simply to e.g., incorrect lexical-stress placement.

With regard to phonological differences between the two languages which could influence accentuation strategy, French has no segmental quantity opposition whereas German has distinctive long and short vowels; French is regarded as a "syllable-timed", German as a "stress-timed" language. These terms, from traditional rhythm typology, are now assumed to cover a number of groups of phonetic and phonological properties that are more or less directly associated with the four prominence-supporting parameters examined here [2, 3, 4]. Dauer's [4] groups are Length, comprising measurable duration, syllable structure and segmental quantity: Pitch, covering the relationship between accentuation and intonation as well as lexical tone; quality, covering the tendency (or not) to reduce vowels and consonants; accent function, which depends on the constraints on lexical stress. Note, however, that the literature seldom makes an explicit distinction between mechanisms for producing syllables of greater and lesser prominence within a word (lexical stress in our terminology) and for accentuating the informational importance of words (phrasal accent). Many instrumental studies conflate the two levels (but see [5]). Indeed, in studies dealing with single word utterances (e.g., [6, 7]), the two levels are inseparable. Here, however, we are looking exclusively at the phonetic exponency of phrasal (de-) accentuation.

\section{Methodology}

\subsection{Material}

As a basis for the direct comparison of parameter values across different levels of phrasal accentuation, utterances with de-accented and accented variants of the same words were needed. Short sentences were constructed containing two one- or two-syllable "critical words" (CWs), one early (but not initial) and one late (but not final) in the sentence. The sentences for German (with the critical words underlined) are:

1. Der Mann fuhr den Wagen vor.

2. Das Bild soll nicht hässlich sein.

3. Das Kind sollte im Bett sein.

4. Der Peter kann den Film gucken.

5. Das Mädchen soll ein Bild malen.

6. Mein Vater kann Türkisch lesen.

The French sentences are:

1. Mon fils met les vases par terre.

2. Mon neveu fait du thé le matin.

3 . Les parcs sont fermés ce soir.

4. Tes pommes sont belles ce matin.

5. Le dentiste s'est cassé la jambe.

6. Mon mari mange du pain ce soir.

For each sentence, questions were devised to elicit a) a broadfocus response, and $\mathrm{b}$ ) a response with a non-contrastive narrow-focus on the early and c) on the late $\mathrm{CW}$.

To provide a (potential) basis for comparing the parameter modification across sentences independently of the differing segmental structuring of the critical words (and thus, if possible, to derive a speakerand/or language-specific quantification of the accent-dependent modification), an iterative "dada" version of each realisation was produced immediately after the normal-text response.

\subsection{Speakers, recordings and analysis}

Six speakers of northern standard French (3f, $3 \mathrm{~m}$ ) and six regionally homogeneous speakers of High German from the Saarland (3f, 3m) produced 6 repetitions of the sentences and their $d a d a$ versions from a PPT presentation in response to the recorded questions. 
Recordings were made in a sound-treated studio on a Tascam DA-P 1 DAT recorder using an AKG C420 ${ }^{\mathrm{III}} \mathrm{PP}$ headset and transferred digitally via the optical channel to a PC using the Kay Elemetrics MultiSpeech speech signal processing program. Segmentation, labelling with SAMPA and further processing was done using the Kiel XASSP speech signal analysis package.

1. Durations were calculated for all feet in the sentences (German feet are left-headed, French feet right-headed), for the $C W \mathrm{~s}$ and their component syllables, and for the other syllables of the feet to which the CWs belonged.. All durational measurements were normalised relative to the mean duration of the corresponding unit in the sentence. Note that this makes the percentage change for the different accent conditions comparable across different size units.

2. Comparisons focussed on changes in identical words across accent levels Therefore, since segmental structure was identical, $\mathrm{F}_{0}$ was calculated as the mean fundamental frequency $(\mathrm{Hz})$ across the syllable nucleus of the lexically stressed syllable of CWs and the unstressed syllables preceding and following them. These values were also normalised by expressing them as a percentages of the mean overall $\mathrm{F}_{0}$ of the sentence.

3. Signal strength was captured in two ways: (i) the normalised mean intensity $(\mathrm{dB})$ of the syllabic nuclei in the lexically stressed syllable of the $\mathrm{CW}$, expressed as a percentage of the mean intensity of the sentence, (ii) the spectral balance (calculated as the difference in intensity between a $70 \mathrm{~Hz}$ to $1 \mathrm{kHz}$ and a 1.2 to 5 $\mathrm{kHz}$ ] frequency band) of the syllabic nuclei in the lexically stressed syllable of the $\mathrm{CW}$. Change as a function of accentual condition was expressed as percent difference from the spectral balance of the broad focus realisation.

4. Spectral definition was captured with the mean frequency values for formants $1-3$ of the syllabic nucleus in the lexically stressed syllable of CWs. Change as a function of accentual condition was expressed for each word as the percentage difference from the broad focus realisation in each formant separately.

\section{Results}

\subsection{Meaningful sentences}

The results for the two languages and the comparison between them are given for each parameter separately. All differences shown in the tables are significant (Tukey post-hoc test together with a multivariate ANOVA) except those which are marked with "!" and highlighted in grey.

\subsection{Duration}

Table 1 gives the overall percentage change in duration between levels of accentuation for different size units.

Table 1. Percentage change in normalized word, syllable and vowel duration between narrow-focus $(\mathrm{Na})$, broad-focus (Br) and de-accented (Da) conditions for French $(\mathrm{Fr})$ and German $(G)$.

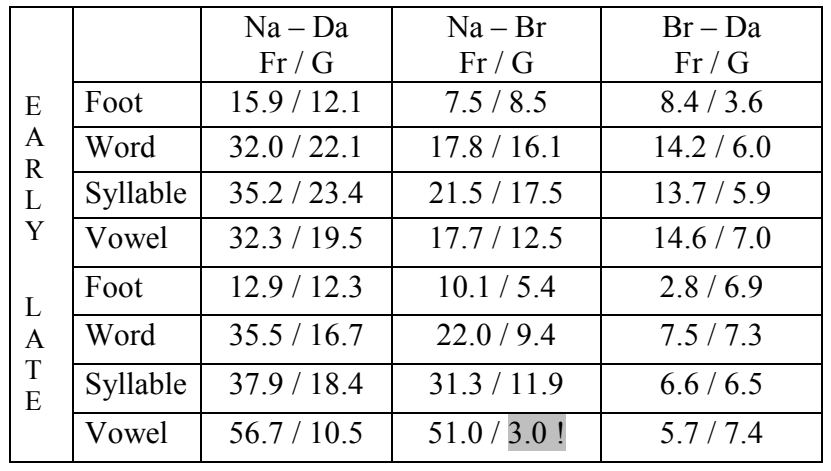

It is apparent that the French speakers exploit durational change for accentuation purposes to a considerably greater degree than the German $(19.8 \%$ more on average across the different units and conditions, cf. column $\mathrm{Na}-\mathrm{Da}$ ). The lower value for the foot unit indicates that the durational change is being borne by the smaller units, and that the foot is not important as a unit of accentuation. In, contrast, both languages show roughly proportionally equal change across the word, syllable and vowel units, in the early position (around 35\% for the French and 20\% for the German speakers), with a slightly greater change at syllable level . In the late position, however, it is the vowel which deviates most from the pattern and which shows the greatest difference between the languages: The French speakers increase the vowel duration by over $50 \%$ for narrow focus accentuation vs. the German speakers' $10 \%$. This suggests that the vowel is the more important accent bearer in French and the syllable overall is more important in German. An alternative interpretation, pending more detailed segmental comparison, is that the syllable rhyme is the accent-bearing unit in both cases - given the assumption that it is the rhyme that defines syllable weight, and given the greater frequency of $\mathrm{CV}$ syllables in French vs. the greater frequency of $\mathrm{CVC}(\mathrm{C})$ syllables in German.

A second important observation from the data in table 1 is the durational differentiation of narrow and broad focus (cf. column $\mathrm{Na}-\mathrm{Br}$ ). Comparison of columns $2(\mathrm{Na}-\mathrm{Br})$ and 3 $(\mathrm{Br}-\mathrm{Da})$ indicate that there is, in fact more difference between the narrow-focus and the broad-focus condition than between broad-focus and the de-accentuated condition, irrespective of whether the $\mathrm{CW}$ bears the nuclear accent (late condition) or not (early).

\subsubsection{Fundamental frequency}

Table 2 shows the percentage difference in the mean normalised $\mathrm{F}_{0}$ for the lexically stressed syllables of the critical words. Table 3 gives the percentage difference in the $\mathrm{F}_{0}$ movement from the preceding (unstressed) syllable to the stressed syllable and from the stressed syllable to the following unstressed syllable (henceforth the $\mathrm{F}_{0}$ "contour").

Table 2. Percentage difference in mean normalised $F_{0}$ for the vowel in the early and late critical syllable between narrow-focus (Na), broad-focus (Br) and de-accented (Da) conditions for French $(F r)$ and German $(G)$

\begin{tabular}{|l|c|c|c|}
\cline { 2 - 4 } \multicolumn{1}{c|}{} & $\begin{array}{c}\mathrm{Na}-\mathrm{Da} \\
\mathrm{Fr} / \mathrm{G}\end{array}$ & $\begin{array}{c}\mathrm{Na}-\mathrm{Br} \\
\mathrm{Fr} / \mathrm{G}\end{array}$ & $\begin{array}{c}\mathrm{Br}-\mathrm{Da} \\
\mathrm{Fr} / \mathrm{G}\end{array}$ \\
\hline Early & $33.1 / 30.2$ & $18.6 / 25.1$ & $14.5 / 5.1$ \\
\hline Late & $39.6 / 37.6$ & $22.6 / 23.0$ & $17.0 / 14.6$ \\
\hline
\end{tabular}

The $\mathrm{F}_{0}$ results correspond to general expectations in that both mean frequency for the syllable and the contour range increase with level of accentuation. With one exception, the two languages differ very little in the manner and degree in which $\mathrm{F}_{0}$ is exploited. Only in the differentiation of early broad-focus and early de-accentuation (table 2, Br-Da column) is there evidence that they behave differently. In contrast to the French, the German speakers show very little difference in mean frequency between these two conditions. The stressed syllable of the early word in broad-focus is only 5\% higher (in relation to the sentence mean F0) than that of the de-accented word.

In terms of contour (table 3 ), the de-accented version is closer to the narrow-focus version than the broad-focus condition. In the early position, the contour for the broad-focus word continues to rise $\left(\mathrm{L}+\mathrm{H}^{*}\right)$, resulting in a negative contour 
value, very different from the step-up $\mathrm{H}^{*}$ accent found in French. In the late position, the frequent down-stepped $\mathrm{H}+\mathrm{H}^{*}$ broad accent results in a continuous fall and consequent low contour value (which differs very little from the flat, postnuclear de-accented contour) compared to the central peak $\mathrm{H}^{*}$ French broad-focus accent.

Table 3. Percentage difference in the $F_{0}$ contour between narrow-focus (Na), broad-focus (Br) and de-accented (Da) conditions for French $(F r)$ and German $(G)$

\begin{tabular}{|l|c|c|c|}
\cline { 2 - 4 } \multicolumn{1}{c|}{} & $\begin{array}{c}\mathrm{Na}-\mathrm{Da} \\
\mathrm{Fr} / \mathrm{G}\end{array}$ & $\begin{array}{c}\mathrm{Na}-\mathrm{Br} \\
\mathrm{Fr} / \mathrm{G}\end{array}$ & $\begin{array}{c}\mathrm{Br}-\mathrm{Da} \\
\mathrm{Fr} / \mathrm{G}\end{array}$ \\
\hline Early & $33.3 / 28.0$ & $22.2 / 30.9$ & $11.1 /-2.9$ \\
\hline Late & $33.9 / 30.4$ & $24.4 / 26.9$ & $9.5 / 3.5$ \\
\hline
\end{tabular}

\subsection{Intensity and spectral balance}

Table 4 gives the percentage difference in intensity $(\mathrm{dB})$ of the critical syllables relative to the overall mean intensity of the sentence, and table 5 shows the corresponding percentage difference in spectral balance calculated for the narrow-focus and the de-accented version relative to the broad-focus condition.

Differences between the languages in mean intensity are minimal. In the early position, the German speakers have a smaller difference than the French between broad-focus and de-accented (though still consistent and significant), whereas their difference between broad-focus and narrow-focus is larger.

Table 4. Percentage difference in mean normalised intensity $(d B)$ of the vowel in the early and late critical syllable between narrow-focus (Na), broad-focus $(\mathrm{Br})$ and deaccented (Da) conditions for French (Fr) and German $(G)$

\begin{tabular}{|l|c|c|c|}
\cline { 2 - 4 } \multicolumn{1}{c|}{} & $\begin{array}{c}\mathrm{Na}-\mathrm{Da} \\
\mathrm{Fr} / \mathrm{G}\end{array}$ & $\begin{array}{c}\mathrm{Na}-\mathrm{Br} \\
\mathrm{Fr} / \mathrm{G}\end{array}$ & $\begin{array}{c}\mathrm{Br}-\mathrm{Da} \\
\mathrm{Fr} / \mathrm{G}\end{array}$ \\
\hline Early & $4.4 / 3.9$ & $2.4 / 3.4$ & $2.0 / 0.5$ \\
\hline Late & $7.3 / 7.5$ & $3.4 / 2.9$ & $3.9 / 4.6$ \\
\hline
\end{tabular}

Table 5. Percentage difference in the spectral balance of the vowel in the early and late critical syllable between narrow-focus (Na), broad-focus (Br) and de-accented (Da) conditions for French $(\mathrm{Fr})$ and German $(G)$

\begin{tabular}{|l|c|c|c|}
\cline { 2 - 4 } \multicolumn{1}{c|}{} & $\begin{array}{c}\mathrm{Na}-\mathrm{Da} \\
\mathrm{Fr} / \mathrm{G}\end{array}$ & $\begin{array}{c}\mathrm{Na}-\mathrm{Br} \\
\mathrm{Fr} / \mathrm{G}\end{array}$ & $\begin{array}{c}\mathrm{Br}-\mathrm{Da} \\
\mathrm{Fr} / \mathrm{G}\end{array}$ \\
\hline Early & $7.8 / 20.1$ & $8.7 / 10.4$ & $-0.9 ! / 9.7$ \\
\hline Late & $17.6 / 13.8$ & $9.4 / 13.5$ & $8.2 / 0.3 !$ \\
\hline
\end{tabular}

The differences in spectral balance between accent levels are, percentage-wise, considerably larger, and the differences between the languages are also clearer. In the early position, the German speakers differentiate much more strongly between the three accent levels, while the French speakers do not differentiate between broad-focus and de-accented (table 5, column $\mathrm{Br}-\mathrm{Da}$ ). This is the result of audible accentual force that remains on the early "de-accented" word. There is no data available to say whether this also occurs in spontaneous French. In the late position, the reverse is found; it is the French speakers who differentiate more strongly and the German speakers who fail to differentiate broad-focus and deaccented. Here, there is no auditory evidence that the (postnuclear) de-accentuation is incomplete, as was observed for French in the early position. It is important to note that these patterns of differentiation do not correlate with the mean intensity changes (compare the relative values in Table 4 and $5)$, underlining reports in the literature $[8,9,5]$ linking spectral balance (or spectral tilt) rather than overall intensity with accentuation.

The reason for the positional difference in the exploitation of spectral balance for accent differentiation is unclear. What is clear is that the two languages do not differ fundamentally in their use of the parameter. Both languages make systematic use of the glottal adjustments that underlie shifts in spectral balance for accentual purposes.

\subsubsection{Vowel quality}

Table 6 gives the percentage difference in Formants 1-3 calculated relative to the broad-focus condition. Based on the rationale that more strongly accentuated implies more extreme, negative values represent formant value differences that go against that assumption.

Table 6. Percentage change in the formant frequencies $(\mathrm{Hz})$ of the vowel in the early and late critical syllable between narrow-focus (Na), broad-focus (Br) and de-accented (Da) conditions for French $(F r)$ and $\operatorname{German}(G)$

\begin{tabular}{|l|c|c|c|c|}
\cline { 3 - 5 } \multicolumn{2}{c|}{} & $\begin{array}{c}\mathrm{Na}-\mathrm{Da} \\
\mathrm{Fr} / \mathrm{G}\end{array}$ & $\begin{array}{c}\mathrm{Na}-\mathrm{Br} \\
\mathrm{Fr} / \mathrm{G}\end{array}$ & $\begin{array}{c}\mathrm{Br}-\mathrm{Da} \\
\mathrm{Fr} / \mathrm{G}\end{array}$ \\
\hline Early & $\mathrm{F} 1$ & $2.8 ! / 8.0$ & $7.0 / 5.2$ & $4.2 ! / 2.8$ \\
\hline & $\mathrm{F} 2$ & $-2.7 / 0.7 !$ & $-0.3 ! / 0.3 !$ & $-2.4 ! / 0.4 !$ \\
\hline & $\mathrm{F} 3$ & $-0.9 ! / 0.3 !$ & $-1.1 ! / 0.4 !$ & $0.2 ! /-0.1 !$ \\
\hline Late & $\mathrm{F} 1$ & $10.8 / 10.8$ & $7.8 / 8.1$ & $3.0 ! / 2.7$ \\
\hline & $\mathrm{F} 2$ & $0.3 ! / 4.1$ & $2.3 / 1.7$ & $-2.6 / 2.4$ \\
\hline & $\mathrm{F} 3$ & $0.5 ! / 1.1$ & $1.5 ! /-0.1 !$ & $-1.0 ! / 1.2$ \\
\hline
\end{tabular}

The expected phonetic shift to a higher F1 with increasing accentuation level (rationale: more time for jaw lowering) is borne out strongly by the German data for both early and late positions (cf. significant positive values). The French data shows the same trend, but the relatively high non-significant difference values reflecting very high variability. This is interesting in view of the lack of long and short vowel-quality differences in the front close to mid-close front region in French compared to German.

The uniform shift in the German data to a higher F2 value with increased accent level (small but significant in late position, non-significant in early position) is plausible, given that five of the six late CWs and four of the early CWs have mid to close front vowels which would naturally manifest higher F2 values if produced with a more extreme quality. The same concentration of front non-open vowels is found in the French sentences $(3 \mathrm{x} / \mathrm{i} /$ and an $/ ø /$ in early position and $3 \mathrm{x} / \mathrm{e} /$, an $/ \varepsilon /$ and $/ \tilde{\varepsilon} /$ in late position) without a corresponding systematic shift in formant values. This underlines the difference between the languages which was found for F1.

\subsection{The iterative "dada" material}

Although the meaningful sentence data supply highly significant main effects and interpretable results which must be considered robust because of the structural variability underlying the CWs, the real extent of any inter-language differences are inevitably clouded. Iterative data was therefore recorded and analysed to supplement the meaningful sentence data. We summarise the results briefly in this section.

Duration: The stronger exploitation of increased duration in French for all units except the foot to reflect accentuation was confirmed. For all units measured, the normalized measures 
were significantly different for all three accentual levels. The following values are restricted to the overall percentage change from de-accented to narrow-focus (compare the $\mathrm{Na}-\mathrm{Da}$ columns in tables 1-6):

$$
\text { Early }
$$

Foot: $\quad$ Fr. 9.9 G. 8.3

Word: Fr. 22.0 G. 15.3

Fr. 9.0 G. 8.7

$\begin{array}{lll}\text { Fr. } 28.8 & \text { G. } 12.5\end{array}$

Syllable: Fr. 25.8 G. 17.0

Fr. 34.2 G. 15.2

Vowel: Fr. 40.9 G. 18.0

Fr. 49.9 G. 12.9

Interestingly, although we have no explanation for it at present, the tendency for French speakers to lengthen accented units more in late position and for German speakers to lengthen the early units more (compare table 1, column Na$\mathrm{Da})$, is also confirmed in the dada material..

Fundamental frequency: There is no a priori reason why the $F_{0}$ patterns for iterative speech should deviate from those of the meaningful sentences. In fact, the French speakers do show remarkably similar difference values both for mean $\mathrm{F}_{0}$ and for $\mathrm{F}_{0}$ contour (compare tables 2 and 3 , column $\mathrm{Na}-\mathrm{Da}$ ). The values for the German speakers are, however, much lower, though they follow the same relative trends: Early Late

$\begin{array}{lllll}\text { Mean } F_{0}: & \text { Fr. } 32.1 & \text { G. } 23.1 & \text { Fr. } 35.1 & \text { G. } 29.5 \\ F_{0} \text { movement: } & \text { Fr. } 33.6 & \text { G. } 18.8 & \text { Fr. } 32.0 & \text { G. } 23.9\end{array}$

Intensity and spectral balance: Since the inherent intensity and the frequency distribution of energy can vary considerably across vowel categories, the dada values should offer a less noisy comparison of French and German.

\begin{tabular}{lllll} 
& \multicolumn{2}{c}{ Early } & \multicolumn{2}{c}{ Late } \\
Intensity $(d B):$ & Fr. 4.4 & G. 4.0 & Fr. 7.2 & G. 7.3 \\
Spectral balance: & Fr. 14.0 & G. 19.3 & Fr. 27.6 & G. 39.3
\end{tabular}

The normalised mean intensity differences are uniform across language, and the pattern of greater differences in late position found in the meaningful sentences is repeated.

The pattern of spectral balance differences is more systematic in the dada material than for the meaningful data. The difference values are greater for the late position in both languages, and the differences are uniformly greater for German than French. Given the variety of vowels in early position in the German meaningful sentences (only two of the six are open central [a] vowels), it is difficult to see why there is no increase in the iterative material (or, alternatively, why the value was so high across the different vowels in the meaningful material.

Vowel quality: Formant value differences between accent levels were expected to become more meaningful in iterative speech. Compare the following values with table 6 , column 1 (F3 has been excluded since none of the differences were significant):

\begin{tabular}{llllll} 
& \multicolumn{3}{c}{ Early } & \multicolumn{3}{c}{ Late } \\
F1: & Fr. 13.7 & G. & \multicolumn{1}{c}{ Fr. } & Fr. 19.3 G. 9.7 \\
F2: & Fr. 5.0 & G. 1.5 & Fr. 4.7 G. 1.5
\end{tabular}

The German values are similar for different vowels in meaningful sentences and the /a:/ in iterative speech. This implies is that the change is purely a function of accent on jaw opening. The much larger, systematic French changes with accent level (contrasting with the unsystematic changes in the meaningful sentences) are due to a clearly audible shift to a less open quality close to $[\mathrm{e}]$ in the de-accented position. There is a clear co-variation with vowel duration (which varies more strongly with accent level in French than in German) which supports the same "universal" implication that was postulated for the German data.

\section{Discussion and Conclusions}

The findings of this study confirm the primary hypothesis that languages will differ systematically in the degree to which they employ the four acoustic dimensions underlying different levels of phrasal accentuation.

The greatest difference between the languages was seen in duration. Words, syllables and vowels are lengthened much more in French than German, supporting the hypothesis that parameters required for other phonological tasks (e.g., long-short vowels in German) are constrained in their use for accentuation. Another difference appears to be the domain of the accentual lengthening: German speakers lengthen the vowel, syllable and word in roughly similar proportions, while the French speakers focus more strongly on the vowel.

As far as $\mathrm{F}_{0}$ is concerned, there is very little difference between the two languages. One difference, however, is in the tonal realisation of the early $\mathrm{CW}$ in the broad-focus. The $\mathrm{L}+\mathrm{H}^{*}$ accent used in German was in contrast to the French $\mathrm{H}^{*}$, and the resulting normalised mean $\mathrm{F}_{0}$ was close to the de-accented value (though the rising tone resulted in a negative contour value which differed from the low positive value found for the deaccented case).

In terms of intensity change with accent, the two languages behave very similarly, but the picture from the spectral balance measures is unclear in the meaningful material. However, the iterative data suggest that German makes greater use of spectral balance differences than French.

German speakers have small but systematic changes in F1 that correlate with accent levels, while the French changes in formant values are similar but irregular in the meaningful sentences and quite large in the iterative material.

The following parameter hierarchy is suggested:

French: Duration $>$ F0 $>$ Spectral balance $>$ F1 $>$ Intensity German: F0 $>$ Duration $>$ Spectral balance $>$ F1 $>$ Intensity

Of course, the hierarchy is purely production orientated, based on normalised acoustic parameters. The perceptual weight of the parameters may be very different.

Acknowledgements: This research was funded by the German Research Council, grant BA 737/10-1.

\section{References}

[1] Peperkamp, S., Dupoux, E., Sebastia'n-Galle's, N. “Perception of stress by French, Spanish, and bilingual subjects," Proc. Eurospeech '99, Vol. 6, 2683-2686, 1999.

[2] Bertinetto P. M. Strutture prosodiche dell'italiano. Firenze: Accademia della Crusca, 1981.

[3] Dauer, R. Stress-timing and syllable-timing reanalyzed. $J$. Phonetics 11: 51-62, 1983.

[4] Dauer, R. Phonetic and phonological components of language rhythm. Proc. XIth ICPhS, vol. 5: 447-450, 1987

[5] Sluijter, A., van Heuven, V. Spectral balance as an acoustic correlate of linguistic stress. J. Acoust. Soc. Amer. 100, 2471-2485, 1996.

[6] Fry, D.B. Experiments in the perception of stress. Lang. \& Speech 1, 126-152, 1958.

[7] Fry, D.B. The dependence of stress judgments on vowel formant structure. Proc. 6th ICPhS Münster, 306-311, 1965.

[8] Kochanski, G., Grabe, E., Coleman, J. Loudness predicts prominence: fundamental frequency lends little. J. Acoust. Soc. Amer. 118, 1038-1054, 2005.

[9] van Kuijk, D., Boves, L. Acoustic characteristics of lexical stress in continuous speech. Sp. Comm. 27, 95-11, 1999. 